\title{
Resonant photodiffractive effect in semi-insulating multiple quantum wells
}

\author{
D. D. Nolte \\ Department of Physics, Purdue University, West Lafayette, Indiana 47907 \\ D. H. Olson, G. E. Doran, W. H. Knox \\ ATET Bell Laboratories, Holmdel, New Jersey 07733
}

A. M. Glass

ATET Bell Laboratories, Murray Hill, New Jersey 07974

Received November 28, 1989; accepted April 10, 1990

\begin{abstract}
We use semi-insulating multiple quantum wells to combine the holographic properties of the photorefractive effect with the large resonant optical nonlinearities of quantum-confined excitons. GaAs-AlGaAs multiplequantum-well structures are made semi-insulating by proton implantation. The implant damage produces defects that are available to trap and store charge during transient holographic recording by means of coherent excitation. The advantages of charge storage and resonant optical nonlinearity combine to produce new optical devices with large sensitivities. The potential use of these devices for image processing is demonstrated by using the Franz-Keldysh effect in four-wave mixing at wavelengths near $830 \mathrm{~nm}$.
\end{abstract}

\section{INTRODUCTION}

The electro-optic properties of multiple quantum wells have been studied extensively ${ }^{1,2}$ and have found application in electro-optic devices. ${ }^{3}$ Such effects and devices rely on the resonant excitation of excitons that remain bound at room temperature by confinement within the quantum-well potentials. These excitons are profoundly affected by perturbations such as free-carrier densities ${ }^{4,5}$ and applied electric fields. ${ }^{6}$ Because of the large oscillator strength of the excitonic absorption and the relatively large influence of small perturbations, large electro-optic and nonlinear-optical effects result. These materials are therefore ideal candidates for use in high-speed digital optical switching and light modulation systems. However, because of the two-dimensional conductivity within the quantum wells, spatially varying optical information cannot be recorded without reticulation of the individual pixels in order to isolate neighboring switching elements. This severely limits the spatial resolution for optical image processing.

Optical materials at the other extreme from electro-optic quantum wells are the bulk photorefractive semiconductors. The photorefractive effect also has been extensively studied, ${ }^{7}$ and many electro-optic materials have found applications in image processing, phase-conjugate mirrors,

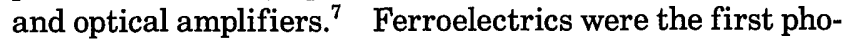
torefractive materials to be studied, because of their large linear electro-optic coefficients. However, ferroelectric applications typically suffer from extremely slow speeds. As an alternative to the slow ferroelectrics, semiconductors have attracted recent interest because of their high mobility and potential speed. ${ }^{8}$ The main attraction of the photorefractive effect is for optical image processing, because spatially varying optical phase or intensity patterns can be recorded and processed with low-intensity light beams.

It would be of significant practical value to combine the large resonant nonlinearities of multiple quantum wells with the parallelism of the photorefractive effect. This has now been done with the use of semi-insulating multiple quantum wells (SIMQW's). In this paper we describe resonant photodiffractive effects in semi-insulating multiple GaAs-AlGaAs quantum wells. The wells are made semi-insulating by proton implantation without adverse effects on the excitonic structure. The essential difference of SIMQW's from conventional conducting quantum wells is the capacity to store charge at the defects introduced by the implant damage. Therefore variation of the electrical potential within the multiple-quantum-well layers can be achieved without reticulation. This permits holographic image storage, as in conventional photorefractive image storage, but with the large resonant optical nonlinearities of multiple quantum wells. We begin with a description of the multiple quantum wells and ion implantation. The electro-optic properties of the SIMQW's are then presented, followed by nonlinear-optical mixing. Diffraction efficiencies from the resonant photodiffractive effect are obtained for various electric fields and are related to the electroabsorption and the electrorefraction.

\section{SEMI-INSULATING QUANTUM WELLS}

In this study GaAs quantum wells with AlGaAs barriers were used to confine room-temperature excitons. The 


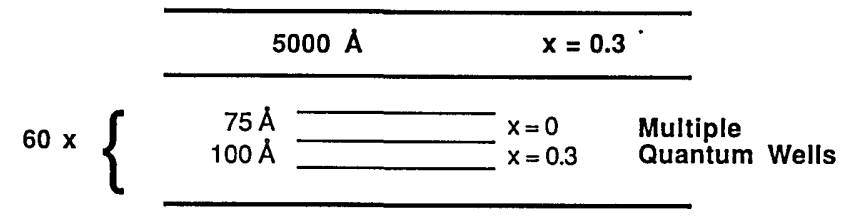

$13000 \mathrm{~A} \quad \mathrm{x}=0.3$

$2000 \mathrm{~A}$ $x=0$

SI GaAs Substrate

Fig. 1. Multiple-quantum-well structure of $\mathrm{GaAs}$ wells with AlGaAs barriers with $30 \% \mathrm{Al}$ fraction.

structure shown in Fig. 1 is a conventional multiplequantum-well structure. An initial epitaxial layer of GaAs $2000 \AA$ thick was grown on a semi-insulating GaAs substrate by using molecular-beam epitaxy. This layer was followed by a $1.3-\mu \mathrm{m}$-thick buffer layer of $\mathrm{Al}_{0.3} \mathrm{Ga}_{0.7} \mathrm{As}$. A total of 60 quantum wells was then deposited. The GaAs well width was $75 \AA$ with $100 \AA$ barriers of $\mathrm{Al}_{0.3} \mathrm{Ga}_{0.7} \mathrm{As}$. A cap of $\mathrm{Al}_{0.3} \mathrm{Ga}_{0.7} \mathrm{As} 5000 \AA$ thick was finally deposited on top of the multiple quantum wells. The active multiplequantum-well region of this structure is $1.05 \mu \mathrm{m}$ thick. The buffer layers provide electrical isolation of the wells from the electrical contacts.

For holographic processing, the conducting multiple quantum wells must be made semi-insulating. In addition the GaAs substrate must be removed to permit transmission of wavelengths appropriate for resonant excitation of the quantum-confined excitons. The layers are made semi-insulating by proton implantation. ${ }^{9}$ After growth the top surface of the multiple-quantum-well structure was implanted with a dose of $10^{12}$ protons $/ \mathrm{cm}^{2}$. The sample was then cleaved into samples with the dimensions $3 \mathrm{~mm} \times 3 \mathrm{~mm}$. The implanted face was epoxied onto a transparent sapphire slide. The exposed substrate was lapped to a thickness of $75 \mu \mathrm{m}$ followed by a selective etch that removed the remaining GaAs down to the AlGaAs buffer layer. This back side was then also implanted with a dose of $10^{12}$ protons $/ \mathrm{cm}^{2}$. The implantation of both the front and the back surfaces was necessary because the implant depth for the protons is approximately $1.5 \mu \mathrm{m}$, compared with the total structure thickness of $2.85 \mu \mathrm{m}$. After implantation the dark resistivity of the multiplequantum-well structure was $10^{9} \Omega-\mathrm{cm}$. This high resistivity is caused by deep implant damage that pins the Fermi-level midgap.

To test what effect the implant damage has on the excitons and the mobility, we measured photoluminescence, absorption, and photoconductivity of the sample. There is a dramatic decrease of the luminescence intensity of the excitons after implantation, which is caused by rapid nonradiative recombination mediated by the implant damage. The room-temperature absorption spectrum is shown in Fig. 2. The heavy- and light-hole excitons remain sharply defined in spite of the damage. The multiple fringes that are visible in the spectrum are FabryPerot fringes from the sample. The heavy-hole excition peaks at $827 \mathrm{~nm}$. The light-hole exciton peaks at $816 \mathrm{~nm}$. The absorption coefficient at the heavy-hole exciton is $\alpha=9000 \mathrm{~cm}^{-1}$, calculated for a thickness of $1.05 \mu \mathrm{m}$, including barriers and wells. The photoconductivity as a function of intensity was measured to be $\sigma_{p} / I=6 \times$ $10^{-3} \mathrm{~cm} / \Omega-\mathrm{W}$. The bipolar mobility-lifetime product of the photocarriers obtained from the photoconductivity data is $\mu \tau=\sigma_{p} h \nu / e I \alpha=1.5 \times 10^{-6} \mathrm{~cm}^{2} / \mathrm{V}$. This mobilitylifetime product compares favorably with normal bulk photorefractive semiconductors in spite of the implant damage. If one assumes a free-electron lifetime of $0.2 \mathrm{nsec}$, the free-carrier density for a pump intensity of $1 \mathrm{~mW} / \mathrm{cm}^{2}$ is approximately $10^{10} \mathrm{~cm}^{-3}$ with a resistivity of $10^{5} \Omega-\mathrm{cm}$. Because the dark resistivity is so high, photoconductivity dominates the conductivity for extremely small laser intensities. This fact contributes to an extremely high recording sensitivity, as will be seen in Section 4.

\section{ELECTRO-OPTIC PROPERTIES}

Measurement of the electro-optic properties of the SIMQW requires contact with the sample to permit application of an electric field. Two geometries are possible, a field parallel to the layers and a field perpendicular to the layers. The two geometries are shown in Fig. 3. In the parallel field geometry the excitons are field ionized by the electric field (Franz-Keldysh effect), leading to lifetime broadening of the exciton peaks. ${ }^{10}$ In the perpendicular geometry the electric field causes the quantum-confined Stark effect, ${ }^{11}$ producing shifts and broadening of the excitons. In our study we have looked only at the parallel geometry with a contact spacing of $1 \mathrm{~mm}$. The changes in the excitonic features with the applied field produce changes in the absorption and the refractive index at wavelengths near the resonant excitation. These electricfield-induced effects are called electroabsorption and electrorefraction, and both effects provide the means by which image gratings can be observed in the material and can be used for optical switching.

The differential transmission spectrum caused by the modulated electric field is shown in Fig. 4 for an electric field of $4 \mathrm{kV} / \mathrm{cm}$ modulated at $150 \mathrm{~Hz}$. For low fields the changes in transmission increase quadratically with electric-field magnitude. The quadratic dependence arises because the electric-field dependence of the excitonic absorption depends not on the sign of the electric field

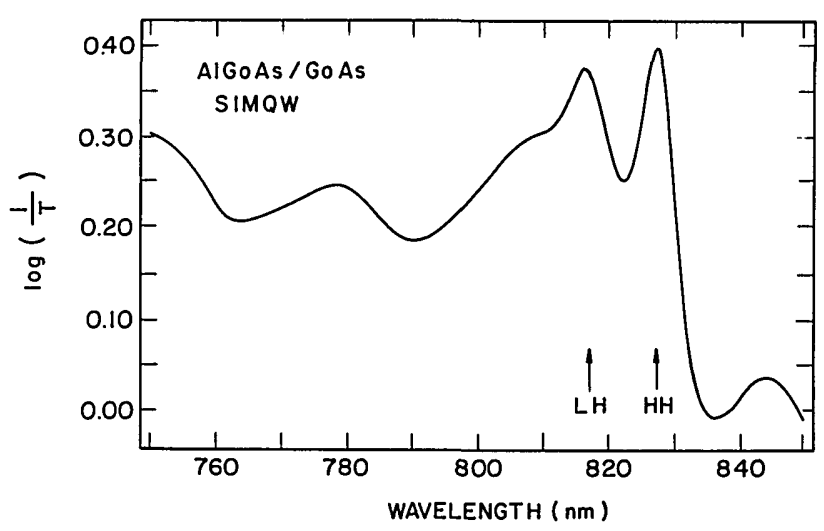

Fig. 2. Absorption in SIMQW after proton implantation. The heavy $(\mathrm{HH})$ and light $(\mathrm{LH})$ holes are not appreciably influenced by the implant damage. The fringe structure is étalons from the sample thickness. 


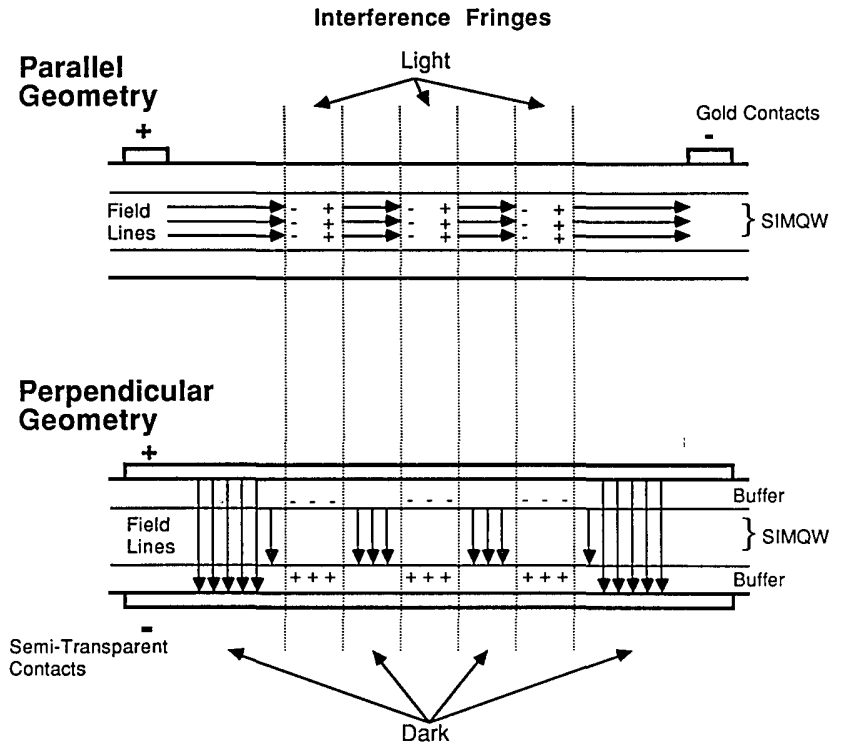

Fig. 3. Device geometries for the SIMQW devices. The parallel geometry studied here yields electroabsorption through the quantum-confined Franz-Keldysh effect. The perpendicular geometry is identical to the self-electro-optic-device geometry that relies on the quantum-confined Stark shift.

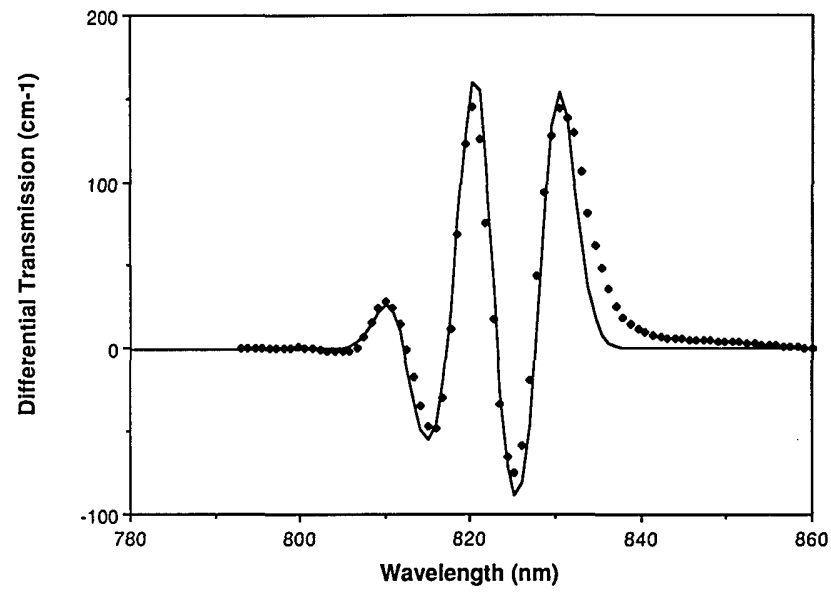

Fig. 4. Differential transmission $\Delta T / L$ for electric-field modulation of $4.2 \mathrm{kV} / \mathrm{cm}$. The solid curve is the fit to the data with the parameters shown in Table 1.

but only on the magnitude. For a discussion of quadratic electro-optic effects, see Ref. 12. The quadratic electroabsorption permits the transmitted light to be detected at twice the modulation frequency, thus eliminating background scatter and any linear excitonic electro-optic effect. The maximum transmission change occurs on the long wavelength side of the heavy-hole exciton. The shape of the spectra is consistent with a broadening of the excitons. No shifts of the excitonic peaks are resolvable.

The differential transmission is used to fit the electroabsorption, $\Delta \alpha(E)=\alpha(E)-\alpha(0)$. The differential transmission is given by

$$
\begin{aligned}
\Delta T & =\exp [-\alpha(E) L]-\exp [-\alpha(0) L] \\
& \approx-\Delta \alpha(E) L \exp [-\alpha(0) L]
\end{aligned}
$$

where $\alpha(0)$ is the zero-field absorption and $\alpha(E)$ is the absorption with an applied electric field. The absorption is fitted by using the procedure of Ref. 1. The functional form of the absorption consists of two Gaussians, one each for the heavy- and the light-hole excitons, and a contribution from the continuum. Nine parameters are required for description of the absorption. These are $\alpha_{h}, \alpha_{l}, \alpha_{c}$ for the magnitudes of the heavy hole, the light hole, and continuum absorptions, $\Omega_{h}, \Omega_{l}, \Omega_{c}$ for the energies of the three features, and $\Gamma_{h}, \Gamma_{l}, \Gamma_{c}$ for the widths of the absorption features. Under an electric field each of these parameters changes. The parameters and their change under $4.2 \mathrm{kV} / \mathrm{cm}$ are given in Table 1 , fitted to the differential transmission data in Fig. 4. The parameters in Table 1 are not unique but give reasonable agreement with the data and with previous studies. ${ }^{1}$ The electroabsorption predicted from these parameters is shown in Fig. 5.

Changes in excitonic absorption are accompanied by changes in the index of refraction. The excitonic electrorefraction can be derived from the excitonic electroabsorption through the Kramers-Kronig transformation

$$
\Delta n(\lambda)=\frac{\lambda^{2}}{2 \pi^{2}} \int_{0}^{\infty} \frac{\Delta \alpha\left(\lambda^{\prime}\right) \mathrm{d} \lambda^{\prime}}{\lambda^{2}-\lambda^{\prime 2}}
$$

This integral is carried out numerically, based on the parameters in Table 1, and covers the range of wavelengths for which the electroabsorption is finite. The resulting change in the refractive index is shown in Fig. 6. The absorption and index changes under an electric field will both contribute gratings that diffract the pump beam.

\section{PHOTODIFFRACTIVE EFFECT AND OPTICAL MIXING}

The photodiffractive effect is the writing of absorption and index gratings in a sample under illumination by two coherent laser beams and the consequent diffraction of radiation into new directions. To describe the unique aspects of the photodiffractive effect in SIMQW's, we begin with a contrasting review of the normal nonresonant photorefractive effect in bulk materials. Gratings are generated in semi-insulating crystals by the intersection of two coherent laser beams inside a sample. The two beams interfere coherently to produce a stationary modulation of intensity $I(x)=I_{0}[1+m \sin (K x)]$, where $I_{0}$ is the intensity of a single beam, $m$ is the modulation amplitude, and $K=2 \pi / \Lambda$ is the spatial frequency with a fringe spacing $\Lambda=\lambda /(2 \sin \theta)$, where $\theta$ is the half-angle between the in-

Table 1. Parameters and Changes for $\Delta E=4.2 \mathrm{kV} / \mathrm{cm}$

\begin{tabular}{cll}
\hline Parameter & $E=0 \mathrm{~V} / \mathrm{cm}$ & $\begin{array}{l}\text { Change in } \\
\text { Parameter }\end{array}$ \\
\hline$\alpha_{h}$ & $9.2 \times 10^{3} \mathrm{~cm}^{-1}$ & $-230 \mathrm{~cm}^{-1}$ \\
$\alpha_{l}$ & $5.3 \times 10^{3} \mathrm{~cm}^{-1}$ & $-120 \mathrm{~cm}^{-1}$ \\
$\alpha_{c}$ & $2.7 \times 10^{3} \mathrm{~cm}^{-1}$ & 0 \\
$\Omega_{h}$ & $1.498 \mathrm{eV}$ & 0 \\
$\Omega_{l}$ & $1.517 \mathrm{eV}$ & 0 \\
$\Omega_{c}$ & $1.520 \mathrm{eV}$ & 0 \\
$\Gamma_{h}$ & $5 \mathrm{meV}$ & $0.20 \mathrm{meV}$ \\
$\Gamma_{l}$ & $5 \mathrm{meV}$ & $0.10 \mathrm{meV}$ \\
$\Gamma_{c}$ & $7 \mathrm{meV}$ & 0 \\
\hline
\end{tabular}




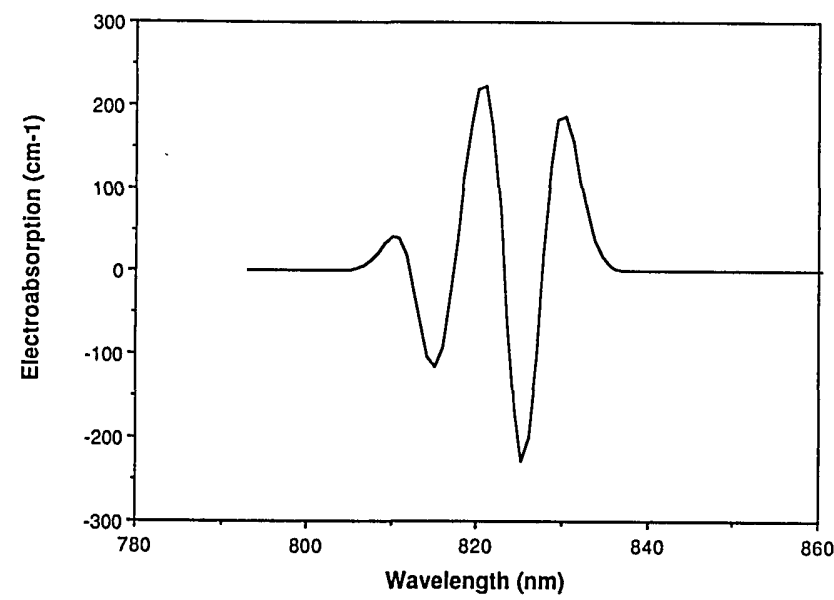

Fig. 5. Electroabsorption for electric field of $4.2 \mathrm{kV} / \mathrm{cm}$, showing change in absorption as a function of wavelength.

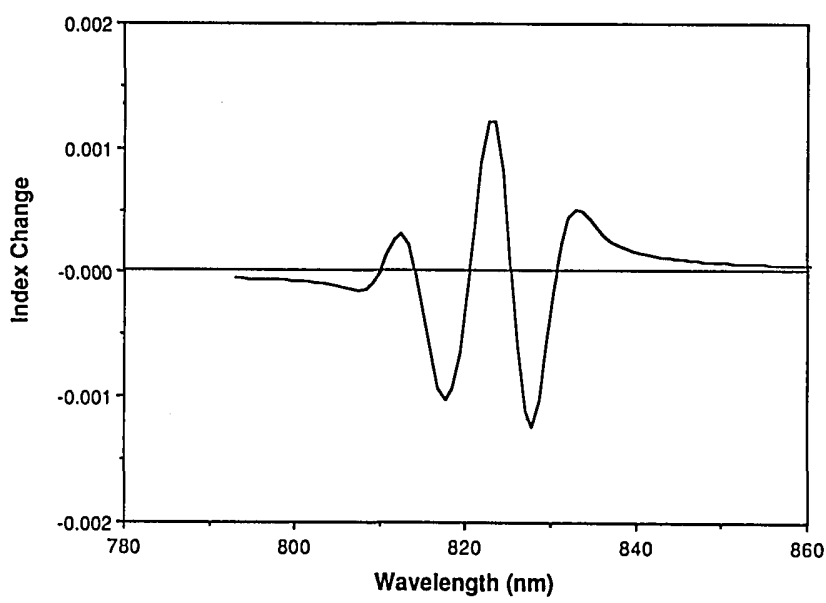

Fig. 6. Electrorefraction for electric field of $4.2 \mathrm{kV} / \mathrm{cm}$, showing change in index of refraction as a function of wavelength.

tersecting beams. For nonresonant illumination, free carriers are photoexcited out of the deep defects that pin the Fermi level. For large applied fields, when diffusion is neglected, the free carriers separate to screen the applied fields and then are retrapped at defect levels in the dark fringes, as shown in Fig. 7. The transport and separation of charge produce internal modulated space charge, with the associated modulated electric field $E_{\mathrm{SC}}$. For the nonresonant case the electric fields modify the index of refraction through the linear electro-optic effect $\Delta n=$ $-1 / 2 n^{3} r_{41} E_{\mathrm{SC}}$, where $n$ is the index of refraction and $r_{41}$ is the linear electro-optic coefficient. The value in GaAs is $r_{41} \approx 1 \mathrm{pm} / \mathrm{V}$. The modulated index of refraction acts as a diffraction grating. A probe beam is diffracted with the efficiency

$$
\eta=e^{-\alpha L} \sin ^{2}\left(\frac{\pi \Delta n L_{\text {int }}}{\lambda \cos \theta}\right)
$$

in a sample of length $L$. The optical interaction length $L_{\text {int }}$ is given by $L_{\text {int }}=\left(1-e^{-\alpha \mathrm{L}}\right) \alpha$. An absorption grating is also generated by the redistribution of charge and also diffracts a probe beam. However, the modulation of the absorption grating by the electric field is negligible in most nonresonant circumstances, so one can usually ignore electroabsorption in the case of nonresonant excitation. It is important to point out that, because the excitation is nonresonant, optical interaction lengths are of the order of several millimeters. The gratings produced are volume holograms. For diffraction to occur the probe beam must be incident at an angle that satisfies the Bragg condition. This constrains the alignment of bulk photorefractive devices.

Photodiffraction in SIMQW occurs in a fashion similar to nonresonant photorefraction, but we highlight the essential differences. For resonant photodiffraction the exciting radiation produces free carriers by generation of electron-hole pairs. As in the photorefractive effect, the free carriers move to screen the external applied fields and are trapped at defects from the implant damage, thus producing a spatially modulated internal electric field. In the case of resonant excitation, electroabsorption can be quite large (in contrast to nonresonant excitation), and absorption gratings can diffract with efficiencies comparable with or larger than diffraction efficiencies of index gratings. Hence we describe resonant diffraction as the photodiffractive effect, which collectively includes nonlinear-optical mixing from electroabsorption as well as from electrorefraction. Because of the large absorption at the band edge, the free-carrier densities in SIMQW's are much larger than those in nonresonant photorefractive materials for the same illumination intensity. Therefore the photoconductivity of the SIMQW's is much larger than that in the nonresonant photorefractive effect, and lower intensities are needed for screening internal fields. In addition, the large electro-optic effects of the excitons makes the diffraction efficiencies from these gratings much larger than those in the nonresonant photorefractive effect, which relies only on the linear electro-optic

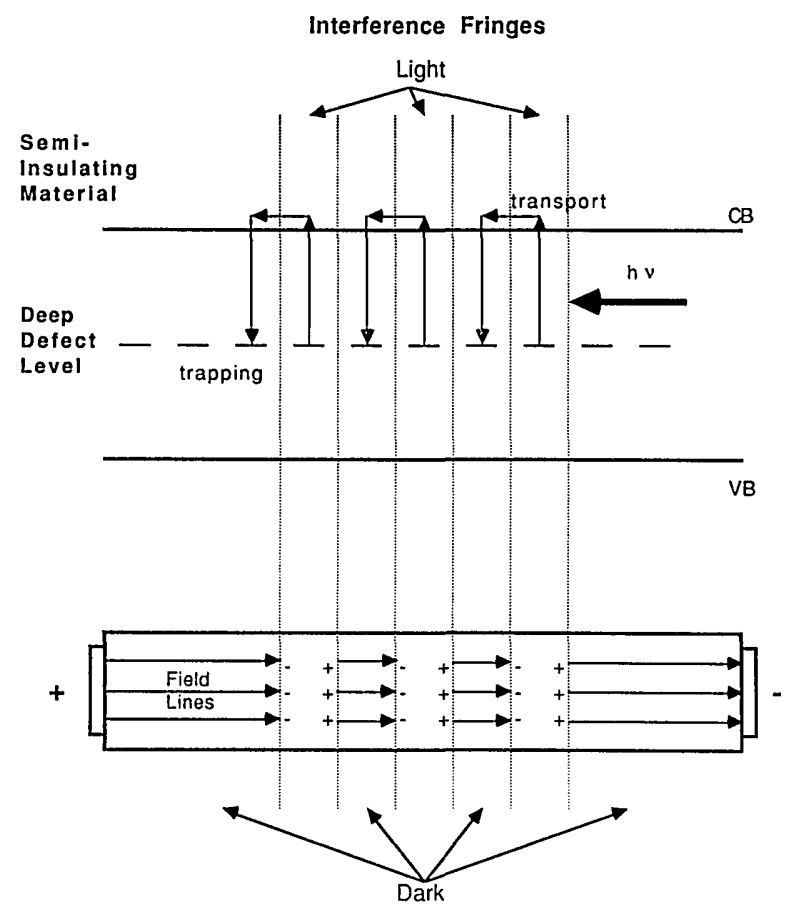

Fig. 7. Photorefractive screening. The carriers generated in the light fringes move to screen the externally applied field. The charge transport produces a spatially modulated space-charge field that yields spatially modulated absorption and index changes. 


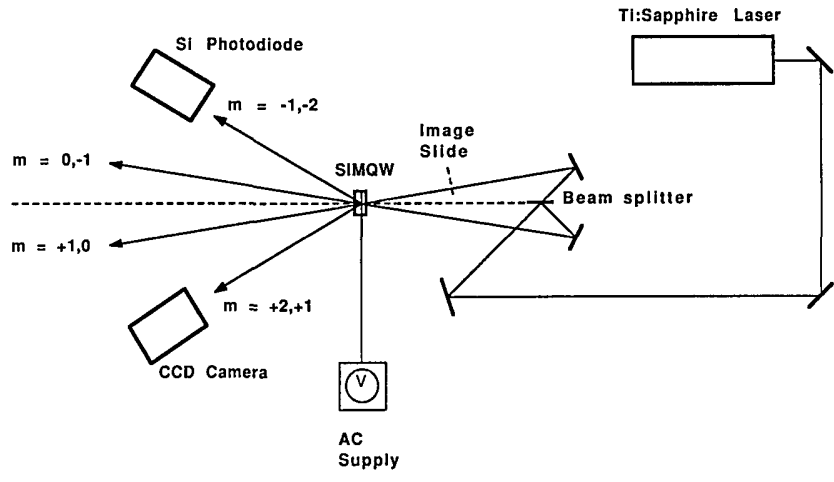

Fig. 8. Mixing geometry with the SIMQW. The beam from the Ti:sapphire laser is split into two beams that interfere coherently inside the sample. The quantum-well thickness is comparable with the grating spacing, which is the criterion for the thingrating limit. Diffraction into higher orders can occur. The diffracted signal is detected in the $m= \pm 1$ order.

coefficient. The large quadratic electro-optic effect in the SIMQW's contributes to large device sensitivity; fewer photons are required for a given index change.

The photodiffractive effect is measured by observation of diffraction from gratings that are written by the interference of coherent laser beams. To provide coherent resonant excitation, we used a Ti:sapphire laser pumped by an Ar-ion laser. The $\mathrm{Ti}$ laser is easily tuned over the wavelength range of interest. The experimental geometry is shown in Fig. 8. The beam from the Ti laser is split by a 50-50 beam splitter into two beams that intersect in the SIMQW sample. The half-angle between the beams is $10^{\circ}$, which leads to a fringe spacing of $2.4 \mu \mathrm{m}$. This fringe spacing is comparable with the thickness of the multiple quantum wells, corresponding to the thin-grating limit, or Raman-Nath diffraction. ${ }^{13,14}$ In this limit diffraction is allowed to higher orders. This is in contrast to the volume holograms of the bulk photorefractive effect, where the Bragg condition must be satisfied. The diffraction gratings in the SIMQW sample therefore diffract each pump beam into the $m= \pm 1$ orders. The diffracted beams are detected by alternating the electric field across the sample and detecting the signal with a Si photodiode at twice the alternating frequency. For reduction of the scattered background, a bandpass filter at $840 \mathrm{~nm}$ was placed in the path of the pump beam, and a 750-nm-long pass filter was placed in front of the $\mathrm{Si}$ detector. Raw spectra therefore contain the spectral dependence of the Ti:sapphire laser combined with the $\mathrm{Si}$ detector response and the transmission functions of the filters.

The raw diffracted signal is shown in Fig. 9 for wavelengths between 820 and $840 \mathrm{~nm}$. The electric field had a rms amplitude of $274 \mathrm{~V}$ and was modulated with a frequency of $170 \mathrm{~Hz}$; the signal was detected at $340 \mathrm{~Hz}$. The pump-beam intensity for this spectrum was $200 \mathrm{~mW} / \mathrm{cm}^{2}$ in each arm. The diffraction efficiency at the peak near $830 \mathrm{~nm}$ was calibrated to be $1 \times 10^{-5}$. The raw electroabsorption is included in Fig. 9 for comparison. An important feature to note is that the diffraction efficiency is large at the zero crossing of the electroabsorption. Therefore the diffracted signal must have a contribution from refractive gratings as well as from absorption gratings.
The absorption and index changes in Figs. 5 and 6 above can be used to understand the qualitative features of the diffracted signal. The total diffraction efficiency for Raman-Nath diffraction is obtained from coupled-mode analysis. ${ }^{13}$ The real and imaginary parts of the index change are related to the real index change and the change in absorption through

$$
\begin{aligned}
\Delta n^{\prime} & =\Delta n+i \Delta k \\
& =\Delta n+i \lambda \Delta a / 4 \pi
\end{aligned}
$$

The total diffraction efficiency to the $m$ th order is given by

$$
\eta_{m}=e^{-\alpha L} J_{m}^{2}\left|\frac{2 \pi \Delta n^{\prime} L_{\text {int }}}{\lambda \cos \theta}\right|,
$$

where $J_{m}$ is a Bessel function of the first kind. This simplifies in the small-signal limit for $m= \pm 1$ to

$$
\eta=e^{-\alpha L}\left[\left(\frac{\pi \Delta n L_{\text {int }}}{\lambda \cos \theta}\right)^{2}+\left(\frac{\Delta a L_{\text {int }}}{4 \cos \theta}\right)^{2}\right] .
$$

The predicted total diffraction efficiency is shown in Fig. 10. The contribution from the absorption grating alone is included.

The dependence of the diffracted signal on the electric field was also investigated. The diffracted signal at $830 \mathrm{~nm}$ is shown in Fig. 11 as a function of the electric field. The signal increases with the fourth power of the field up to $3 \mathrm{kV} / \mathrm{cm}$. Above this field the signal begins to saturate, reaching a maximum near $4 \mathrm{kV} / \mathrm{cm}$. The origin of the saturation is not understood at present. Further study is clearly needed to resolve this question.

The perpendicular geometry should be superior to the parallel geometry because the electro-optic interaction is based on the quantum-confined Stark effect, which is stronger than the Franz-Keldysh effect. In this geometry, shown in Fig. 3, the electric fields are perpendicular to the gratings. In the illuminated fringes the photogenerated charge separates and is trapped at defects in the

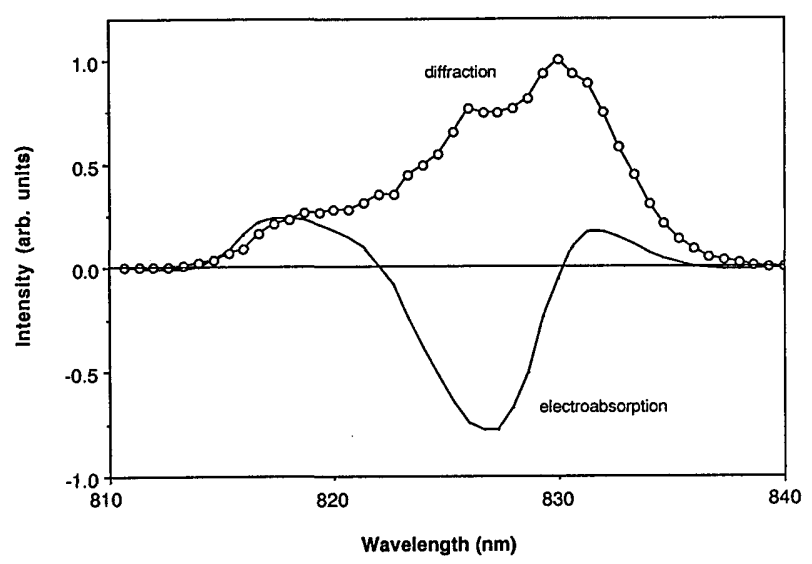

Fig. 9. Experimental diffraction efficiency and associated differential transmission. These data are not normalized. The peak in the diffraction efficiency occurs near the zero crossing of the electroabsorption. This indicates that index gratings play a significant role in the diffraction. The peak diffraction efficiency is $1 \times 10^{-5}$. 


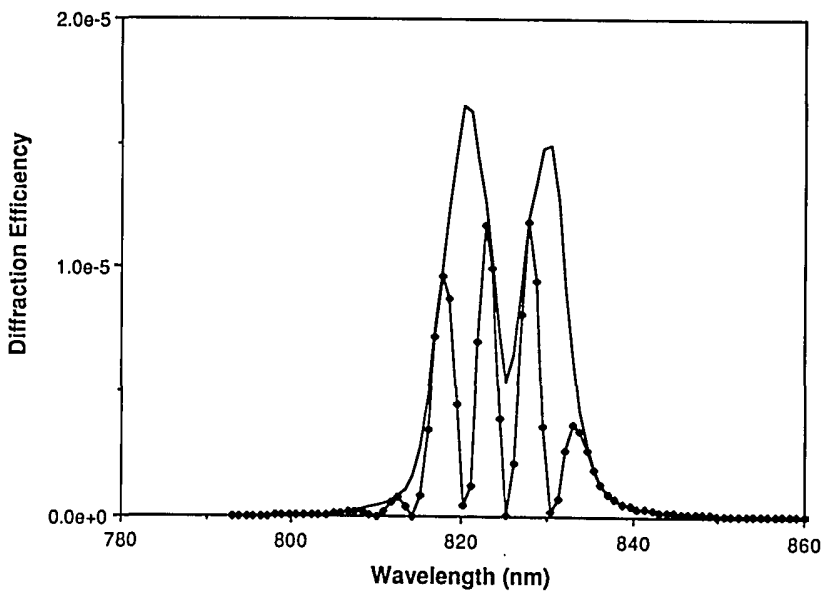

Fig. 10. Predicted diffraction efficiency from the fit of Fig. 4 The total diffraction efficiency from both the absorption and the index gratings is given by the solid curve. The contribution from the absorption grating alone is shown by the curve that connects the points.

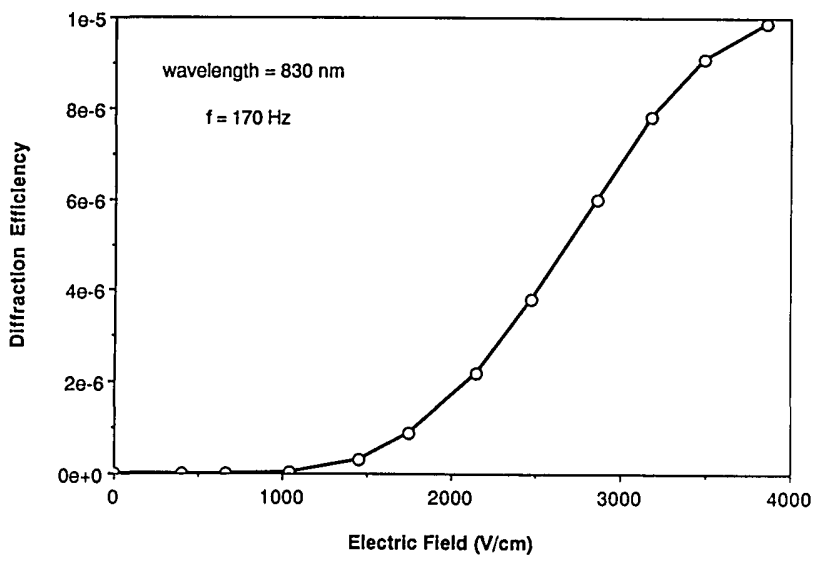

Fig. 11. Diffraction efficiency as a function of electric field. The efficiency increases with the fourth power of the field at low field strength.

semi-insulating AlGaAs buffer layers, screening out the applied fields. In the dark fringes the applied electric field will not be screened, and the excitonic absorption will be altered by the quantum-confined Stark effect. For maximum spatial electric-field modulation (and maximum diffraction efficiency) the multiple-quantum-well thickness cannot be much larger than the fringe spacing; otherwise fringing fields will wash out the spatial electricfield modulation. Maximum absorption changes, comparable with the absorption changes in self-electro-optic devices, ${ }^{3}$ can be expected, leading to diffraction efficiencies of several percent with only $1 \mu \mathrm{m}$ of multiple-quantumwell material.

\section{DEVICE PERFORMANCE}

For facilitation of comparisons among different materials and different device geometries, it is useful to establish figures of merit that are related to general device requirements. Many figures of merit exist, each highlighting the specific ability of a device for a specific function, such as maximum index change per photon, minimum saturation intensity, and device speed. The figures of merit can be constructed from only a few parameters that were discussed in the previous sections. The material parameters are the mobility-lifetime product, $\mu \tau=10^{-6} \mathrm{~cm}^{2} / \mathrm{V}$ the absorption, $\alpha=10^{4} \mathrm{~cm}^{-1}$; and the dark resistivity, $\rho_{d}=10^{9} \Omega$-cm. The device parameters are: the maximum index change, $\Delta n=0.001$; the electric field for maximum index change, $E=4 \mathrm{kV} / \mathrm{cm}$; and the fringe spacing, $\Lambda=2.4 \mu \mathrm{m}$. With these parameters six figures of merit are constructed: saturation intensity $I_{\text {sat }}$ (also called the dark intensity, $I_{d}$ ), maximum internal field $E_{\max }$, device speed $\tau_{D}$, switching energy $E_{\mathrm{SW}}$, recording sensitivity $S^{\prime}$, and the $\Delta n L$ product.

The saturation intensity $I_{\text {sat }}$ is the lowest pump-laser intensity that saturates the diffraction efficiency. This is the intensity for which the photoconductivity equals the dark conductivity, i.e., $\sigma_{p}=\sigma_{d}$. For intensities below $I_{\text {sat }}$, the dielectric relaxation rate is faster than the photocarrier production rate, and insufficient charge density can be accumulated to screen the internal fields fully. For low pump intensities, the diffraction efficiency increases linearly with the pump intensity. At higher intensities, when the photoproduction rate of free carriers becomes greater than the relaxation rate, the diffraction efficiency saturates to a constant value for a constant electric field. At the lowest detectable intensity in our experiment, $400 \mu \mathrm{W} / \mathrm{cm}^{2}$, the signal was already saturated. The saturation intensity can be calculated by finding the intensity for which the photoconductivity is equal to the dark conductivity:

$$
I_{\text {sat }}=\frac{\sigma_{d} h \nu}{\mu \tau \alpha e}=100 \mathrm{nW} / \mathrm{cm}^{2} .
$$

This saturation intensity is $10^{4}$ times smaller than in nonresonant photorefractive materials. This factor comes from $\alpha=10^{4} \mathrm{~cm}^{-1}$ for the quantum wells, compared with $\alpha \approx 1 \mathrm{~cm}^{-1}$ for nonresonant bulk material. Much higher carrier densities are produced for the same intensity.

The maximum internal field modulation $E_{\max }$ is limited by the number of defects available to trap charge and thereby screen externally applied fields. For a trap density $N_{d}$, the maximum field is

$$
E_{\max }=\frac{e N_{d} \Lambda}{2 \pi \varepsilon \varepsilon_{0}} .
$$

External fields larger than this cannot be screened entirely because sufficient space charge cannot be developed. In the proton-implanted material, for a dose of $10^{12} \mathrm{~cm}^{-2}$, the defect density was estimated to be $10^{17}$ $10^{18} \mathrm{~cm}^{-3}$. Such high defect densities yield $E_{\max }=$ $5 \times 10^{5}$ to $E_{\max }=5 \times 10^{6} \mathrm{~V} / \mathrm{cm}$, which is larger than externally applied fields. The defect density should therefore be sufficient for total screening in any practical circumstances.

The device speed is set by the response time $\tau_{R}$, which is the time required for sufficient charge transfer to occur in order to screen the internal fields. At zero field this is simply the dielectric relaxation time ${ }^{15}$

$$
\tau_{\mathrm{di}}=\frac{\varepsilon \varepsilon_{0}}{\sigma_{d}+\sigma_{p}}
$$


The relaxation time for $I_{\mathrm{sat}}$ is $\tau_{\mathrm{sat}}=1 \mathrm{msec}$. For increased intensity the relaxation time can be expressed as

$$
\tau_{\mathrm{di}}=1 \mathrm{msec}\left(\frac{I_{\mathrm{sat}}}{I}\right) .
$$

For an intensity of $100 \mathrm{~mW} / \mathrm{cm}^{2}$ this value corresponds to a response time of 1 nsec. In nonresonant undoped GaAs for the same intensity, the relaxation time is $10 \mu \mathrm{sec}$. Again, the large absorption coefficient leads to much larger conductivity and therefore to much faster devices.

The response time is modified in the presence of electric fields. For a given field, enough time is required so that the full charge transfer is permitted to screen the field. The larger the field, the more charge is necessary and the more time is needed. Therefore the response time increases with an increased field. The charge integration time $t_{I}$ is set by the charge transfer rate $\mathrm{d} N / \mathrm{d} t$, and the charge density $N_{E}$ needed to screen a field $E$ is given by

$$
\frac{\mathrm{d} N}{\mathrm{~d} t} t_{I}=N_{E}=\frac{2 \pi \varepsilon \varepsilon_{0} E}{e \Lambda} .
$$

The charge transfer rate is determined by the production rate of free carriers and by the probability that the carriers are trapped within the first fringe spacing, given by $t_{t} / \tau$, where $t_{t}=1 / K \mu E$ :

$$
\begin{aligned}
\frac{\mathrm{d} N}{\mathrm{~d} t} & =\frac{I \alpha}{h \nu} \frac{t_{t}}{\tau} \\
& =\frac{I \alpha}{h \nu} \frac{\Lambda}{2 \pi \mu \tau E} .
\end{aligned}
$$

The charge integration time is therefore given by

$$
\begin{aligned}
t_{I} & =\tau_{\mathrm{di}}\left(\frac{2 \pi \mu \tau E}{\Lambda}\right)^{2} \\
& =\tau_{\mathrm{di}}\left(\frac{2 \pi l_{d}}{\Lambda}\right)^{2},
\end{aligned}
$$

where $l_{d}$ is the drift length. The device response time $t_{\mathrm{R}}$ is obtained by combining the charge integration time with the dielectric relaxation time to yield

$$
\tau_{R}=\tau_{\mathrm{di}}+t_{I},
$$

where diffusion has been neglected. The dependence of the device relaxation time on the square of the electric field greatly decreases the speed of the device. This is especially significant for the high fields that will be necessary to achieve reasonable diffraction efficiencies. For a field of $10^{4} \mathrm{~V} / \mathrm{cm}$ and an intensity of $100 \mathrm{~mW} / \mathrm{cm}^{2}$ the device response time is $\tau_{R}=100 \mu \mathrm{sec}$, which is $10^{5}$ times slower than the zero-field response. The charge integration time is applicable to nonresonant photorefractive materials as well as to these SIMQW's. The advantage of $10^{4}$ from the quantum-well absorption is still present in the case of an applied field. The SIMQW devices therefore remain significantly faster than nonresonant devices for the same applied fields.

The switching energy $E_{\mathrm{SW}}$ is defined by the energy that is incident upon the device during the time required to switch the device at its highest speed (set by the device response time). The switching energy is

$$
\begin{aligned}
E_{\text {sw }} & =I \tau_{R} . \\
& =5.8\left[1+\left(\frac{2 \pi \mu \tau E}{\Lambda}\right)^{2}\right] \frac{\mathrm{aJ}}{\mathrm{bit}},
\end{aligned}
$$

where aJ stands for attojoule $\left(=1 \times 10 \mathrm{~cm}^{-18} \mathrm{~J}\right)$ and the bit size is set by the size of the fringe spacing: $1 \mathrm{bit}=$ $5.8 \mu \mathrm{m}^{2}$. For a field of $10^{4} \mathrm{~V} / \mathrm{cm}$, the switching energy is $E_{\mathrm{SW}}=900 \mathrm{fJ} /$ bit.

The device sensitivity $S^{1}$ is defined as the index change per absorbed photon:

$$
\begin{aligned}
S^{\prime} & =\frac{\Delta n}{\alpha I \tau_{R}} \\
& =\frac{\Delta n}{\alpha E_{\mathrm{sw}}} \\
& =\frac{e \mu \tau \Delta n}{\varepsilon \varepsilon_{0} h \nu}\left[1+\left(\frac{2 \pi \mu \tau E}{\Lambda}\right)^{2}\right]^{-1},
\end{aligned}
$$

where $h \nu=1.5 \mathrm{eV}$ and we have neglected dark conductivity. The sensitivity saturates quickly to $S^{\prime}=10^{-2} \mathrm{~cm}^{3} / \mathrm{J}$, independent of the field. The quadratic increase of $\Delta n$ with the field is matched by the quadratic increase of the device response time with the field. It is important to note that Eq. (16) is also valid for nonresonant photorefractive effects in GaAs. All parameters are the same for SIMQW's as they are for the bulk except $\Delta n$. The resonant optical nonlinearities of the quantum wells can be much greater than the linear electro-optic effect. The enhancement of the resonant quadratic electro-optic effect over the nonresonant linear electro-optic effect is

$$
\begin{aligned}
\frac{\Delta n_{\text {res }}}{\Delta n_{\text {nonres }}} & =\frac{s}{r_{41}} E \\
& =2 \frac{\mathrm{cm}}{\mathrm{V}} E,
\end{aligned}
$$

where $\Delta n_{\text {res }}=-(1 / 2) n^{3} s E^{2}$ and $s\left(=2 \times 10^{-12} \mathrm{~cm}^{2} / \mathrm{V}^{2}\right)$ is the quadratic electro-optic coefficient at the wavelength of the heavy-hole exciton. This value for the quadratic coefficient is obtained from our measurements of the resonant diffraction efficiency at the zero crossing in the electroabsorption. A field of $10^{4} \mathrm{~V} / \mathrm{cm}$ gives a $10^{4} \mathrm{en}$ hancement of the device index change over those of nonresonant devices.

To produce a device with a specific diffraction efficiency, the relevant figure of merit is the product of the index change times the device length $\Delta n L$. This product enters directly into Eq. (6), the expression for diffraction efficiency. For an optimal device the device length is equal to the absorption depth, i.e., $L=1 / \alpha$. This product at resonance for the parallel geometry device is

$$
\Delta n L=\frac{\Delta n}{\alpha}=10^{-7} \mathrm{~cm}\left(\frac{E}{4 \mathrm{kV} / \mathrm{cm}}\right)^{2}
$$

which is the same as that for nonresonant GaAs for the same grating period. The $10^{4}$ increase in the index change is counterbalanced by the $10^{4}$ decrease of device length. 
This is why the diffraction efficiency for the SIMQW's is $10^{-5}$, comparable with that for bulk GaAs. However, if the pump excitation is detuned slightly from resonance, then the product can be increased. The absorption falls off exponentially with photon energy detuning, but the index change falls off much more slowly. ${ }^{12,16}$ The $\Delta n L$ product can therefore be increased by detuning from resonance. Phonon interactions prevent the sudden fall-off of absorption ${ }^{17}$ that would enable the $\Delta n L$ product to increase exponentially. This product is also limited by the quantum-well thickness $L$, which is typically $1 \mu \mathrm{m}$. The possibility of stacking several thin-grating devices in order to obtain a device in the Bragg regime has been suggested $^{18}$ and may provide a means of increasing the interaction length. Resonant enhancement of photorefractive gain can be achieved in bulk semiconductors by tuning closely enough to the band-edge absorption. ${ }^{19}$

\section{SPATIAL LIGHT MODULATION}

The primary feature that distinguishes SIMQW's from conventional conducting quantum wells is the ability to store charge spatially. This storage capability enables images to be recorded in the material and to be read out or switched into one of the higher diffraction orders. In conducting quantum wells this image recording cannot occur under cw, low-intensity illumination because the dielectric relaxation time is too short; the conductivity erases any charge separation. It is still possible to use free-carrier gratings in short-pulse, high-intensity (lowsensitivity) operation. ${ }^{4,5,20}$ This mode is not ideal for many image-processing applications. The great advantage of image processing is the massive parallelism. Images can consist of megabits of information. For such an image, a switching time as slow as $1 \mathrm{msec}$ still yields a datum rate of $1 \mathrm{Gbit} / \mathrm{sec}$. High speed is therefore not necessary for image processing. The high sensitivity of the SIMQW's is also an important feature if image processing is to occur without high-intensity lasers or without image amplification.

To test the image-switching properties of our SIMQW sample, we placed large and small circular objects with diameters of approximately $1 \mathrm{~mm}$ in the path of one of the two pump beams. A charge-coupled-device (CCD) camera, attached to a video display, was focused through the sample and onto the images of the circles on the glass slide. In the $m=0$ order, the direct undiffracted image of the CCD screen had little optical distortion. To prevent saturation of the CCD, neutral-density filters were placed in the main pump beam before the beam splitter. For detection of the diffracted image in the $m=+1$ order, the $4-\mathrm{kV} / \mathrm{cm}$ electric field was modulated with a frequency near $20 \mathrm{~Hz}$. This produced a clearly discernible flicker of the diffracted image at $40 \mathrm{~Hz}$. The CCD objective lens in this case was focused again through the sample, but this time to the virtual position of the diffracted image. The large and small circles were clearly observable in the diffracted image. However, the quality of the images was considerably reduced compared with that of the direct image. The images of the circles included Fresnel rings. The image quality may have been a result of internal reflections that produced multiple gratings in the sample. These internal reflections may be reduced by depositing antireflection coatings on the sample surfaces. Further investigation of diffracted image quality is necessary.

\section{CONCLUSIONS}

In conclusion, we combined the charge-storage capability of semi-insulating material with the large resonant optical nonlinearities of multiple quantum wells. The combination of the photodiffractive effect with quantum-confined excitons was carried out in GaAs-AlGaAs multiple quantum wells that were made semi-insulating by proton implantation. The implant damage produces highresistivity material with many defects that are available to trap and store charge during a holographic recording. The recording mechanism in the semi-insulating multiple quantum wells is directly analogous to the recording mechanism in the standard nonresonant photorefractive - effect. Free carriers that are generated by photoexcitation by the coherent pump-laser beams screen externally applied electric fields. The coherent interference spatially modulates the laser intensity, and therefore modulates the electric fields. These fields act on the optical properties of the quantum confined excitons, producing quadratic electrorefraction and electroabsorption. The spatial modulation of the fields therefore produces spatial modulation of the index and absorption, or diffraction gratings, which are observed as diffraction of the pump beams into higher orders.

The SIMQW's have good image-storage sensitivities. By going to resonant conditions, the index change per photon is much larger than that for nonresonant excitation. First, the quadratic excitonic electro-optic effects can be much larger (at sufficiently large fields) than the linear electro-optic effects in the photorefractive semiconductors. Therefore, for the same charge displacement as that produced by an absorbed photon, the index change is much larger in the resonant case. Second, the large absorption of the band edge produces a higher density of free carriers (which move to screen external fields) than in the nonresonant case. Therefore a much lower intensity is required for modulation of the field. This enhanced sensitivity is not surprising. It has been well established that resonant excitation is the most successful method for generating large optical nonlinearities. With the combination of photorefractive behavior with this resonant excitation, the advantages of both join to form a promising new genre of devices that may find uses in diffraction-limited image processing, spatial light modulation, holographic storage, and optical switching.

\section{ACKNOWLEDGMENTS}

We are grateful to D. S. Chemla, A. White, and C. Tu for helpful contributions.

\section{REFERENCES}

1. D. S. Chemla, D. A. B. Miller, P. W. Smith, A. C. Gossard, and W. Wiegmann, IEEE J. Quantum Eloctron. QE-20, 265 (1984).

2. D. A. B. Miller, J. S. Weiner, and D. S. Chemla, IEEE J. Quantum Electron. QE-22, 1816 (1986). 
3. D. A. B. Miller, D. S. Chemla, T. C. Damen, T. H. Wood, C. A. Burrus, A. C. Gossard, and W. Wiegmann, IEEE J. Quantum Electron. QE-21, 1462 (1985).

4. J. Hegarty, M. D. Sturge, A. C. Gossard, and W. Wiegmann, Appl. Phys. Lett. 40, 132 (1982).

5. D. A. B. Miller, D. S. Chemla, D. J. Eilenberger, P. W. Smith, A. C. Gossard, and W. Wiegmann, Appl. Phys. Lett. 42, 925 (1983).

6. D. A. B. Miller, D. S. Chemla, T. C. Damen, A. C. Gossard, W. Wiegmann, T. H. Wood, and C. A. Burrus, Phys. Rev. B 32, 1043 (1985).

7. P. Gunter and J. P. Hignard, eds., Photorefractive Materials and Their Applications (Springer-Verlag, Berlin, 1988), Vols. 1 and 2.

8. A. M. Glass, A. M. Johnson, D. H. Olson, W. Simpson, and A. A. Ballman, Appl. Phys. Lett. 44, 948 (1984).

9. Y. Silberberg, P. W. Smith, D. A. B. Miller, B. Tell, A. C. Gossard, and W. Wiegmann, Appl. Phys. Lett. 46, 701 (1985).

10. F. L. Lederman and J. D. Dow, Phys. Rev. B 13, 1633 (1976).
11. D. A. B. Miller, D. S. Chemla, T. C. Damen, A. C. Gossard, W. Wiegmann, T. H. Wood, and C. A. Burrus, Phys. Rev. Lett. 53, 2173 (1984).

12. M. Glick, F. K. Reinhart, G. Weimann, and W. Schlapp, Appl. Phys. Lett. 48, 989 (1986); J. S. Weiner, D. A. B. Miller, and D. S. Chemla, Appl. Phys. Lett. 50, 842 (1987).

13. A. Yariv and P. Yeh, Optical Waves in Crystals (Wiley, New York, 1984).

14. H. Kogelnik, Bell. Syst. Tech. J. 48, 2909 (1969).

15. See G. C. Valley and J. F. Lam, in Ref. 7, p. 75.

16. A. Alping and L. A. Coldren, J. Appl. Phys. 61, 2430 (1987).

17. A. Von Lehman, J. E. Zucker, J. P. Heritage, and D. S. Chemla, Phys. Rev. B 35, 6479 (1987).

18. R. V. Johnson and A. R. Tanguay, Jr., Opt. Lett. 13, 139 (1988).

19. A. Partovi, A. Kost, E. M. Garmire, G. C. Valley, and M. B. Klein, Appl. Phys. Lett. 56, 1089 (1990).

20. R. K. Jain and M. B. Klein, Appl. Phys. Lett. 35, 454 (1979). 